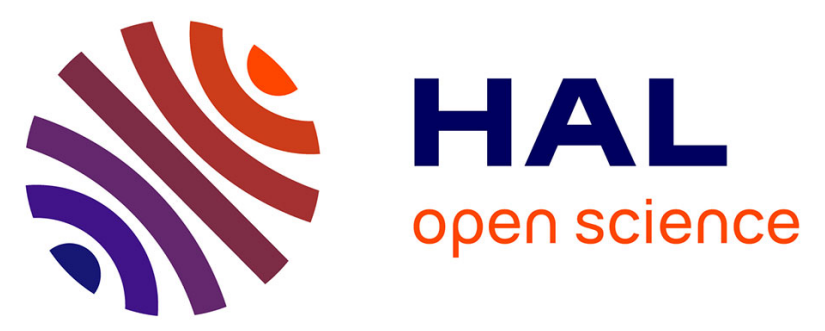

\title{
Detection of Moroccan Coastal Upwelling Fronts in SST Images using the Microcanonical Multiscale Formalism
}

\author{
Ayoub Tamim, Hussein Yahia, Khalid Daoudi, Khalid Minaoui, Abderrahman
} Atillah, Driss Aboutajdine, Mohammed Faouzi Smiej

\section{- To cite this version:}

Ayoub Tamim, Hussein Yahia, Khalid Daoudi, Khalid Minaoui, Abderrahman Atillah, et al.. Detection of Moroccan Coastal Upwelling Fronts in SST Images using the Microcanonical Multiscale Formalism. Pattern Recognition Letters, 2015, 55, pp.5. 10.1016/j.patrec.2014.12.006 . hal-01120898

\section{HAL Id: hal-01120898 \\ https://hal.inria.fr/hal-01120898}

Submitted on 24 Mar 2015

HAL is a multi-disciplinary open access archive for the deposit and dissemination of scientific research documents, whether they are published or not. The documents may come from teaching and research institutions in France or abroad, or from public or private research centers.
L'archive ouverte pluridisciplinaire HAL, est destinée au dépôt et à la diffusion de documents scientifiques de niveau recherche, publiés ou non, émanant des établissements d'enseignement et de recherche français ou étrangers, des laboratoires publics ou privés. 


\title{
Detection of Moroccan Coastal Upwelling Fronts in SST Images using the Microcanonical Multiscale Formalism
}

\author{
Ayoub Tamima,**, Hussein Yahia ${ }^{\mathrm{b}}$, Khalid Daoudi $^{\mathrm{b}}$, Khalid Minaoui ${ }^{\mathrm{a}}$, Abderrahman Atillah $^{\mathrm{c}}$, Driss Aboutajdine ${ }^{\mathrm{a}}$, Mohammed \\ Faouzi Smiej ${ }^{\mathrm{c}}$ \\ ${ }^{a}$ Mohammed V-Agdal University, LRIT Associated Unit to the CNRST-URAC n ${ }^{\circ} 29$, Rabat, Morocco \\ ${ }^{b}$ INRIA Bordeaux Sud-Ouest (GEOSTAT team), Talence, France \\ ${ }^{c}$ Royal Centre for Remote Sensing (CRTS), Rabat, Morocco
}

\begin{abstract}
Nonlinear signal processing using the Microcanonical Multiscale Formalism (MMF) is used to the problem of detecting and extracting the upwelling fronts in coastal region of Morocco using Sea Surface Temperature (SST) satellite images. The algorithm makes use of the Singularity Exponents (SE), computed in a microcanonical framework, to detect and analyze the critical transitions in oceanographic satellite data. The objective of the proposed study is to develop a helpful preprocessor that transforms SST images into clean and simple line drawing of upwelling fronts as an input to a subsequent step in the analysis of SST images of the ocean. The method is validated by an oceanographer and it is shown to be superior to that of an automatic algorithm commonly used to locate edges in satellite oceanographic images. The proposed approach is applied over a collection of 92 SST images, covering the southern Moroccan Atlantic coast of the years 2006 and 2007. The results indicate that the approach is promising and reliable for a wide variety of oceanographic conditions.
\end{abstract}

\section{Introduction}

The sea surface temperature (SST) images obtained from Advanced Very High Resolution Radiometer (AVHRR) sensor have allowed the oceanographers to study and monitor the mesoscale features in the oceans (Holyer and Peckinpaugh. 1989). On SST images, mesoscale structures are delimited by thermal fronts that occur between two water masses of different temperatures; they may be often associated to a convergence zone which is shown to contain loci of high biological activity (Bakun 2006). As a consequence, the knowledge of thermal fronts is an important factor for the fish management and aggregation (Chassot et al. 2011).

In coastal upwelling systems, the upwelling fronts and filaments are perfect examples of such thermal structures, which are, on top of SST data, evidenced by the presence of low temperature values and high chlorophyll concentration in onshore water.

\footnotetext{
** Corresponding author at: Mohammed V-Agdal University, LRIT Associated Unit to the CNRST-URAC n ${ }^{\circ} 29$, Rabat, Morocco. Tel.: +212 655686150; e-mail: ayoubtamim@gmail. com (Ayoub Tamim)
}

A variety of techniques and algorithms have addressed the problem of automated detection of upwelling fronts in SST images. The straightforward and the most popular methods used to distinguish these structures, are the conventional horizontal gradients computed from classical convolution operators (Belkin and O'Reilly, 2009), based on the fact that upwelling fronts are characterized by a very complex horizontal alteration in the upper ocean, generating strong thermal gradients. In this context, Oram et al. (2008) developed an edge detection algorithm specifically for detecting features in sea surface satellite images. The latter is started with the application of gradient based edge detection using the Canny edge detector with an automatic selection of the upper and lower thresholds values from the cumulative global histogram calculated for each image. Then series of steps (edge following, edge validation and edge merging) are used to accurately and objectively detect the upwelling fronts in satellite images of the ocean. Another class of methods deals directly with the gray-level histogram based on the idea that edges in SST images are usually regions of separation between two regions of constant temperatures $(\mathrm{Ni}-$ eto et al. 2012). Therefore an edge is considered to be present if the algorithm detects two significantly different populations. 
Another method that combines the histogram based separation and structure growing process has also been applied to satellite images (Marcello et al. 2005), where the bimodality in an SST image is used to detect the first approximation of the upwelling fringe separating the cold waters near the coast and warmer offshore waters followed by the structure growing process in order to attain the fine detail of the upwelling structures.

The foremost shortcoming encountered in the preceding approaches, is that they have been constructed for use other than the oceanographic context, and consequently they often work poorly when used to detect the mesoscale structures in ocean satellite images (Cayula and Cornillon, 1992). The conventional methods used in image processing often rely on simple hypotheses, compared to the physical of the phenomena encountered. In particular, an SST image is a measure of a thermodynamical variable, the temperature of the ocean's microscopic upper layer in a turbulent flow (the surface of the ocean); the existence of turbulence implies the formation of extremely complex structures that conventional (linear) approaches cannot apprehend, mainly because of the absence of localization in the Fourier transform. As a matter of fact, the intermittency and the existence of multiscale organization present in Fully Developed Turbulence (FDT) (Frisch, 1995) make the upwelling region a complex system relevant to nonlinear signal processing.

In Isern-Fontanet et al. (2007), the authors use the Microcanonical Multifractal Formalism (MMF) and the Most Singular Manifold (MSM) in order to reconstruct the stream function from a specific reconstruction formula defined by a universal kernel propagator applied to satellite data. The resulting stream function is then used to derive mesoscale ocean dynamics. While the authors make use of the singularity exponents and the MSM to determine mesoscale ocean dynamics, the work proposed in our study focuses on the thermal fronts detection from the knowledge of the singularity exponents. Additionally, the thermal fronts detected are validated by a professional oceanographer using a database of 92 SST images of Moroccan atlantic coast. The present paper is part of a larger effort to test the feasibility and the relevance of the Microcanonical Multifractal Formalism to the Moroccan coastal upwelling. In particular, we show that edge pixels computed in such a complex systems framework provides superior validated results in the case of oceanographic data.

The flowchart of the algorithm is presented in Fig. 1, and the remainder of the paper is organized as follows. Section 2 presents the database and the geographic area of study used in this work. Section 3 describes the method used for the automatic detection and extraction of upwelling fronts in SST images. Section 4 reports the results conducted to evaluate the proposed approach. The conclusion and the future work are reported in Section 5.

\section{Study Area and Data}

In this study, we make use of a database of 92 AVHRR SST images covering the southern part of Moroccan Atlantic coast $\left(20^{\circ} 0^{\prime}-27^{\circ} 60^{\prime} \mathrm{N}\right.$ and $\left.13^{\circ} 35^{\prime}-23^{\circ} 30^{\prime} \mathrm{W}\right)$ during the years 2006 and 2007. This region, belonging to the Canary upwelling system, is characterized by strong and persistent thermal structures

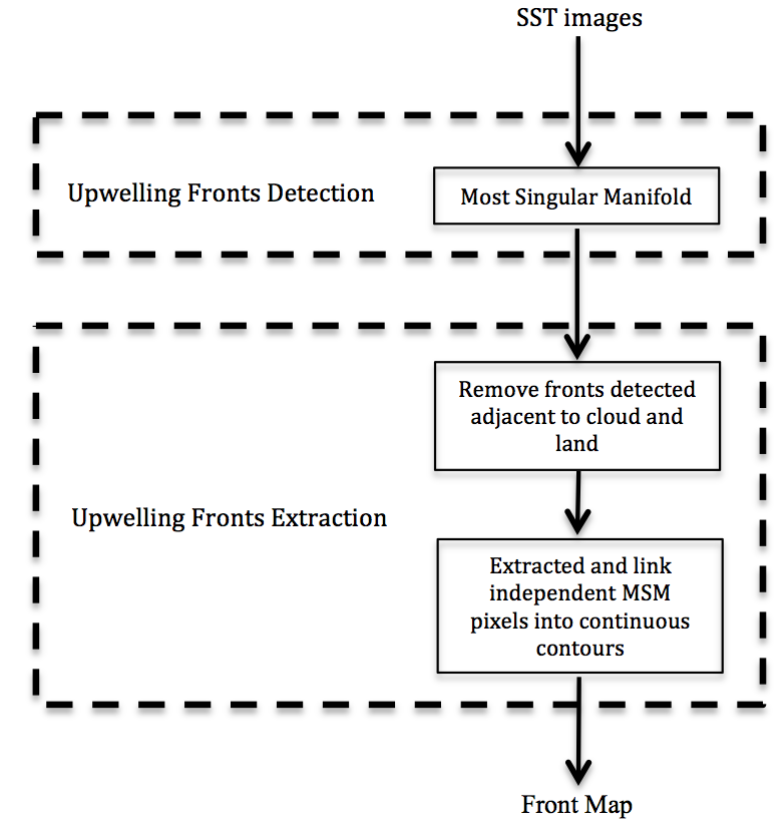

Fig. 1: Simplified block diagram of the automatic algorithm used to detect the thermal upwelling structures in SST images.

(Atillah et al. 2005), including upwelling fronts which separate the cold coastal waters and warmer offshore oceanic waters, and also include the filaments which, represent the extensions of upwelling fronts in open ocean.

The SST images used in this study are received and processed at the Royal Centre of Remote Sensing (CRTS) of Morocco, including geometric, radiometric and atmospheric corrections and also include the cloud and land masks. The cloud overlay is generated using the multispectral radiance measurements (Stowe et al. 1999) tested and evaluated over the AVHRR satellite images. The size of each SST image is $770 \times 990$ pixels, with spatial resolution of $1.1 \times 1.1 \mathrm{~km}$. The temperatures, in Celsius degrees, are estimated using the non-linear SST algorithm (NLSST) (Walton et al. 1998), which greatly reduces any dependence of the gamma parameter upon the satellite measurements and has been shown to be more accurate than the linear algorithm, Multi-Channel SST (MCSST) (McClain et al. 1985).

Due to the a priori knowledge of our region of interest and the fact that the coastal upwelling is largely conditioned by the width and the direction of the continental shelf (Atillah et al. 2005), we explore the whole database and retain for our analysis only SST images that have at least $50 \%$ of valid information (no clouds or missing pixels) in the region of interest (ROI) between the coast and $200 \mathrm{~km}$ offshore (Nieto et al. 2012). Additionally, this ROI is chosen to be parallel to the coastline direction ( $N E-$ $S W$ ), in order to efficiently explore the effect of the width of Moroccan continental shelf (Atillah et al. 2005, Metzner and Hennings. 1995). The dates of the 92 SST images selected from the years 2006 and 2007 are detailed in Table 1 .

Fig. 2 shows two SST images selected from our benchmark of 92 SST images, illustrating two scenarios of upwelling variability: well-defined upwelling in terms of relatively strong 


\begin{tabular}{|c|c|c|c|c|c|c|c|c|c|c|c|c|}
\hline Years & Jan & Feb & Mar & Apr & May & Jun & Jul & Aug & Sep & Oct & Nov & Dec \\
\hline 200 & $\begin{array}{l}06, \quad 14, \\
24,28\end{array}$ & $\begin{array}{l}03, \quad 16, \\
25\end{array}$ & $\begin{array}{l}05, \quad 13, \\
15,25\end{array}$ & $\begin{array}{l}02, \quad 07 \\
22,25\end{array}$ & $\begin{array}{l}04, \quad 15, \\
22\end{array}$ & $\begin{array}{ll}01, & 07, \\
17, & 23, \\
30 & \end{array}$ & $\begin{array}{l}07, \quad 19, \\
27,31\end{array}$ & $\begin{array}{l}11, \quad 19, \\
21,31\end{array}$ & $\begin{array}{l}10,20, \\
25\end{array}$ & $\begin{array}{l}01, \quad 10, \\
19,24\end{array}$ & $\begin{array}{l}02, \quad 14, \\
24,26\end{array}$ & $\begin{array}{l}06, \quad 15 \\
26,29\end{array}$ \\
\hline 2007 & $\begin{array}{l}02, \quad 14, \\
20,30\end{array}$ & $\begin{array}{l}09, \quad 14, \\
22\end{array}$ & $\begin{array}{l}02, \quad 12, \\
14,22\end{array}$ & $\begin{array}{l}04, \quad 14 \\
22,27\end{array}$ & $\begin{array}{l}08, \quad 11 \\
19,30\end{array}$ & $\begin{array}{l}08, \quad 16 \\
19,28\end{array}$ & $\begin{array}{l}07, \quad 15, \\
22\end{array}$ & $\begin{array}{l}03, \quad 05 \\
16,25\end{array}$ & $\begin{array}{l}04, \quad 11, \\
14,24\end{array}$ & $\begin{array}{l}03, \quad 12 \\
21,29\end{array}$ & $\begin{array}{l}01, \quad 09, \\
17\end{array}$ & $\begin{array}{l}02, \quad 03, \\
17,26\end{array}$ \\
\hline
\end{tabular}

Table 1: Dates of the 92 SST images used in this study, covering the years 2006 and 2007.

thermal fronts and with continuity along the coast (Fig. 2(a)), and upwelling with weak thermal gradients and where the cloud contamination affects the continuity of upwelling along the coast (Fig. 2 (b)). These two images will be used throughout this paper to illustrate the validity of our proposed algorithm.

\section{Fronts detection methodology}

\subsection{Fronts detection}

Transitions of the graylevel values inside complex natural images, such that SST images, are related to the existence of a hierarchy of multiscale structures characteristic of FDT (Frisch. 1995). Singularities in SST images corresponding to sets of pixels with sharp and strong variations in intensities values, unlock the multiscale organization (Turiel et al. 2008b). In this work we use a novel formalism called the Microcanonical Multifractal Formalism (MMF) for the analysis of these singularities, based on the strength of variations around any given pixel, which yields to a precise description of upwelling fronts in SST images.

For an SST image $I$ defined by its graylevel function $I(\vec{x})$ and its norm of gradient $\|\nabla I\|(\vec{x})$ at each pixel $\vec{x}$, we introduce a measure $\mu$ defined by its density with respect to the Lebesgue measure, which corresponds to summing the norm of the gradient:

$$
d \mu(\vec{x})=d \vec{x}\|\nabla I\|(\vec{x}),
$$

So that for a ball $\mathcal{B}_{r}(\vec{x})$ of radius $r$ centered around the pixel $\vec{x}$, the measure $\mu$ is given by:

$$
\mu\left(\mathcal{B}_{r}(\vec{x})\right)=\int_{B_{r}(\vec{x})} d \vec{x}\|\nabla I\|(\vec{x})
$$

We work with a normalized luminance intensities :

$$
I(\vec{x})=I(\vec{x})-I_{0}, \quad I_{0}=\int_{\Omega} I(\vec{x}) d \vec{x},
$$

$(\Omega$ : image's domain) such that, the luminance function $I(\vec{x})$ has zero average.

The measure $\mu$ defined above is a multifractal measure, and for any pixel $\vec{x}$ over a set of fine scales $r$ the following equality holds :

$$
\mu\left(\mathcal{B}_{r}(\vec{x})\right)=\alpha(\vec{x}) r^{d+h(\vec{x})}+o\left(r^{d+h(\vec{x})}\right) \quad(r \rightarrow 0)
$$

where $d=2$ indicates the dimension of space ( $d=2$ for an image), and the $\alpha(\vec{x})$ is a signal-dependent amplitude prefactor.

The exponent $h(\vec{x})$ defined in equation (4) is the singularity exponent (SE) at $\vec{x}$ (Turiel et al. 2006); it quantifies the strength of the SST image at the pixel $\vec{x}$ in a statistically coherent way across the scales.

It is worth mentioning that the MMF is not relying on "thermalized" values associated to large ensembles averages and ergodic hypotheses; it has been shown that such "canonical" approaches show difficulties in the processing of empirical data (Turiel et al. 2006); instead, in a microcanonical analogy, the behaviour of the measure is evaluated locally and geometrically at every given pixel location, without relying on thermalized averages (Maji and Yahia, 2014), it has been shown that such an approach is computationally more efficient and, most importantly, it gives access to the geometric localization of transition fronts.

A central concern in MMF is the proper determination, at high numerical precision, of the singularity exponents $h(\vec{x})$. In this context, an approach presented in (Turiel et al., 2008a) (Pont et al., 2013) provides numerically stable computation of the SE at each pixel $\vec{x}$, based on the wavelet projection of the measure:

$$
h(\vec{x})=\frac{\log \left(\tau_{\psi} \mu\left(\vec{x}, r_{0}\right)\right) /\left\langle\tau_{\psi} \mu\left(., r_{0}\right)\right\rangle}{\log r_{0}}+o\left(\frac{1}{\log r_{0}}\right)
$$

where $\tau_{\psi} \mu\left(\vec{x}, r_{0}\right)$ is a projection of measure $\mu$ evaluated at point $\vec{x}$ and scale $r_{0}$ with wavelet $\psi$ (not necessarily admissible, as positive mother wavelets display interesting computational properties) and $\left\langle\tau_{\psi} \mu\left(., r_{0}\right)\right\rangle$ is the average value of the wavelet projection over the whole signal, with $r_{0}$ chosen to reduce the relative amplitude of the correction term $o\left(\frac{1}{\log r_{0}}\right)$. The scale $r_{0}$ is chosen such that a discretized image of size $N \times M$ corresponds to size 1 , so that one chooses $r_{0}=\frac{1}{\sqrt{N \times M}}$.

It is expected that the set of pixels having the strongest transitions, i.e. the least values of singularity exponents, also known as the Most Singular Manifold (MSM) contains most of a turbulent's signal information and provides indications in the natural image about the pixels where sharp and sudden local variations take place. Moreover, from a complex systems approach, the MSM is made of most unpredictable points. The MSM is defined as:

$$
\mathcal{F}_{\infty}=\left\{\vec{x}: h(\vec{x})=h_{\infty}=\min (h(\vec{x}))\right\}
$$

Visual inspection of the MSM pixels in natural images reveals the presence of the contours and edges. Fig. 3 a) shows the set of MSM pixels, corresponding to the smallest values of singularities exponents calculated over the original image in Fig. 2(a).

\subsection{Fronts extraction}

The presence of cloud contamination in the input image (pixels with gray color on the left side of Fig. 22a) and (b)) can have 


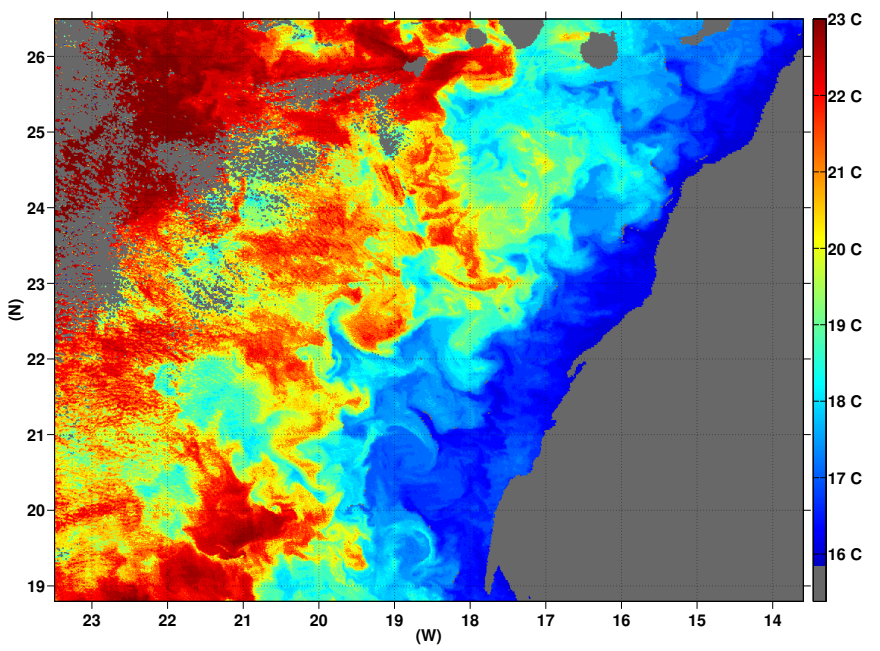

(a)

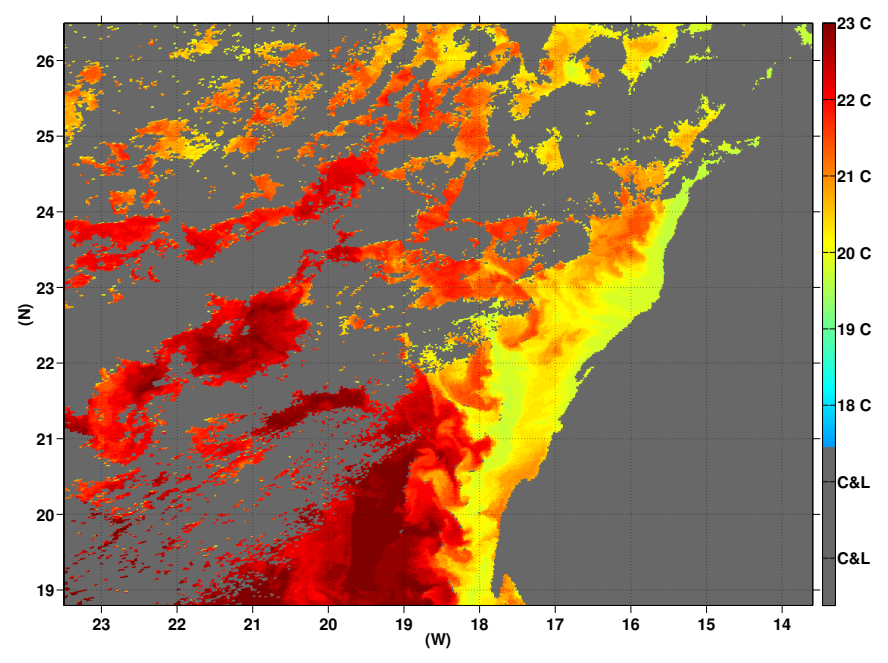

(b)

Fig. 2: SST images obtained respectively on (a) 2007-01-02 and (b) 2006-01-06, showing different upwelling scenarios.

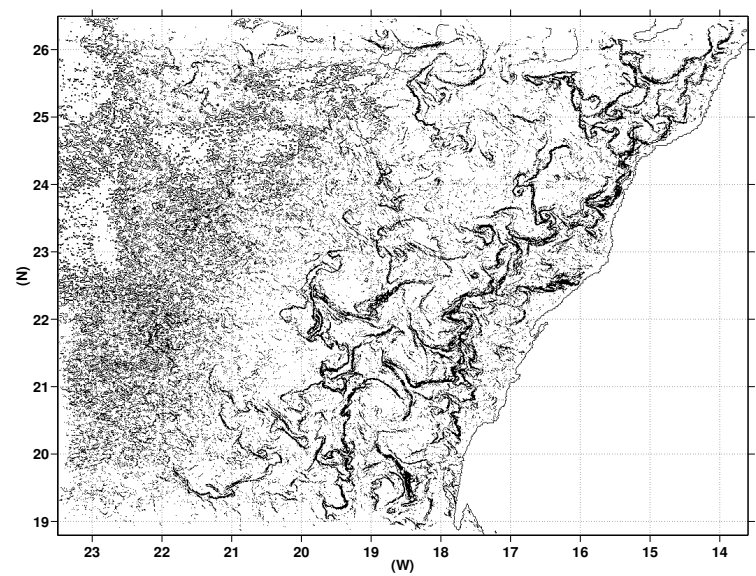

(a)

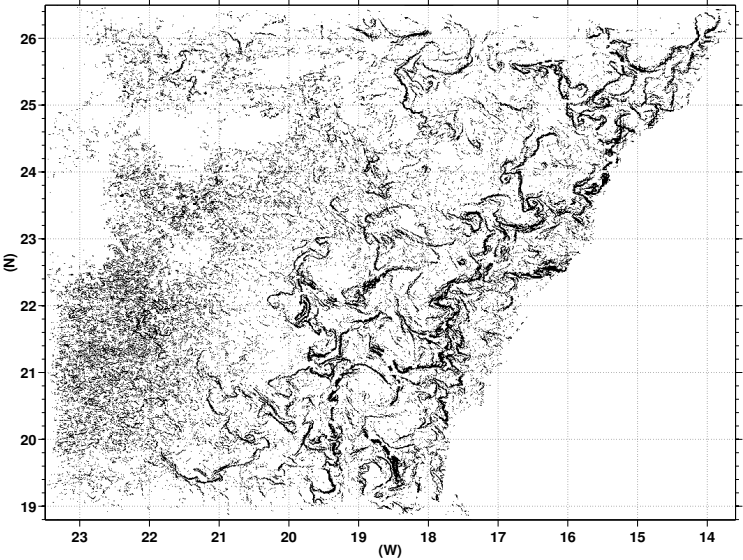

(b)

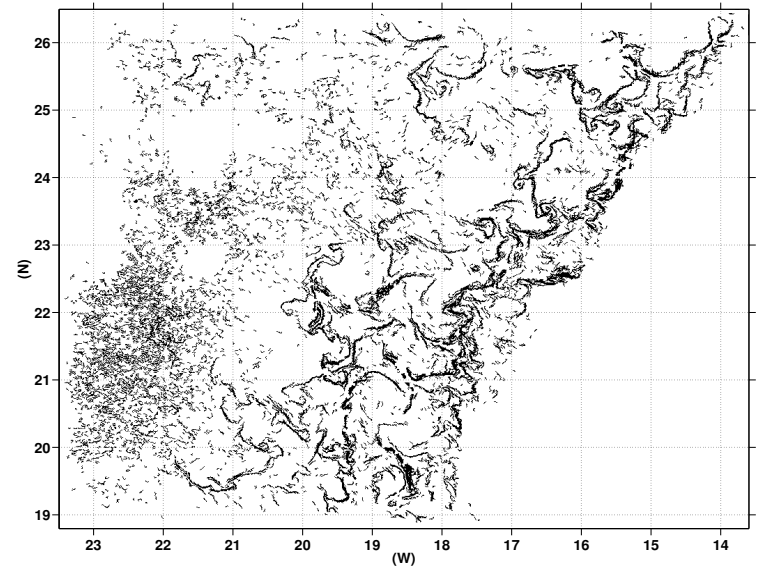

(c)

Fig. 3: Edge maps after localization and detection of upwelling fronts in the original images of Fig.22a): (a) MSM points corresponding to $20 \%$ pixel density, (b) MSM map after removing all the pixels adjacent to cloud and land, and (c) final results of the MSM detection. 


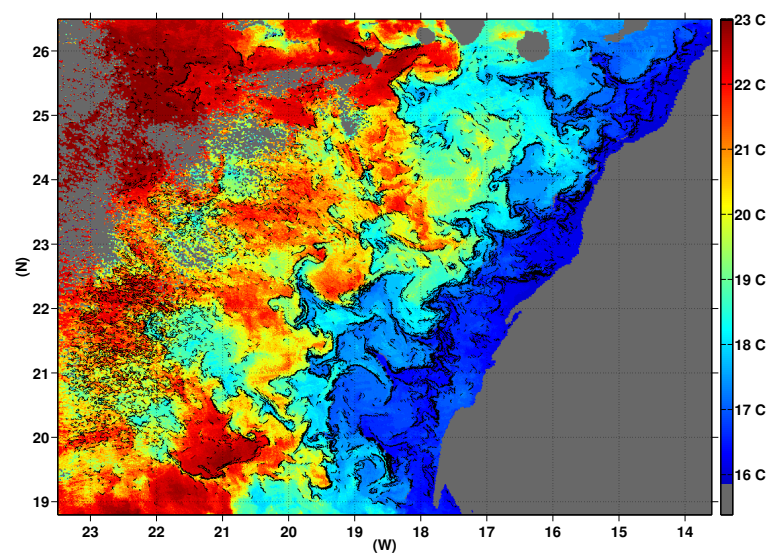

(a)

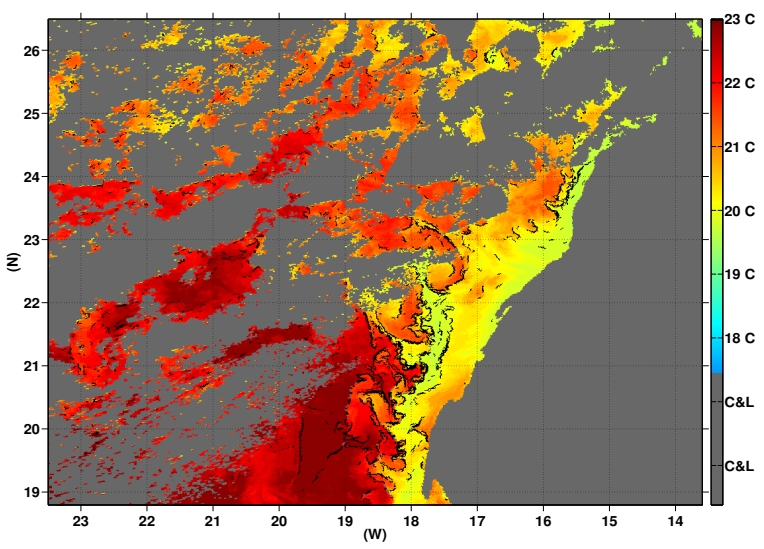

(b)

Fig. 4: (a) Fronts shown in Fig. 3 c) overlaid on the original image in Fig. 2.a); (b) Fronts detected in the same way (MSM method) overlaid on the original SST image in Fig. 2b).

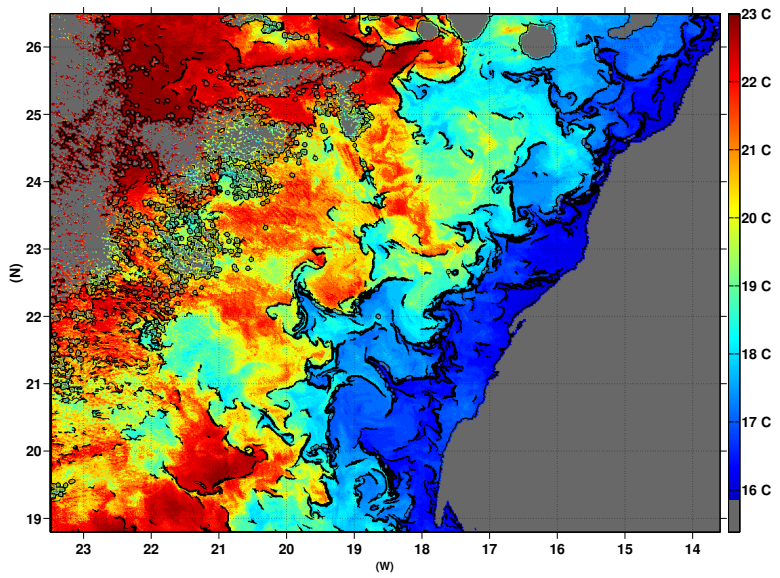

(a)

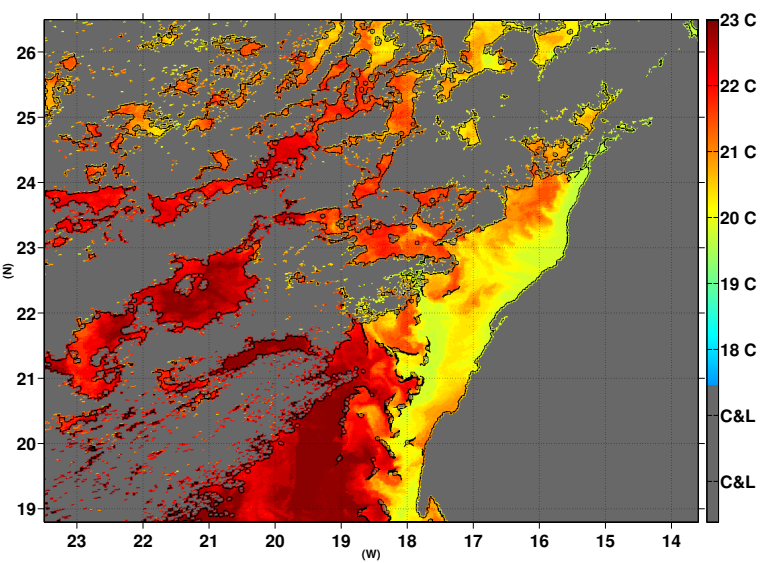

(b)

Fig. 5: Results of applying the algorithm Oram et al. (2008) respectively to the original images in Fig. 2. a) and (b). 
serious effects on the results of edge pixel detection. Indeed, the MSM points detected in Fig. 3 (a) include the MSM pixels of upwelling fronts separating cold and warmer water, and the MSM pixels of cloud fronts separating the cloud and water masses.

To avoid the misclassification of the cloud frontal boundaries as upwelling fronts, we used the cloud mask cited in Stowe et al. (1999), such that for each MSM pixel in the Fig. 3 a), if the 8-neighboring pixels contain at least one cloud pixel, the MSM of the given pixel is discarded. Fig. 3 (b) shows the MSM map after removing all the MSM pixels adjacent to clouds in Fig. 3. a).

In order to examine each individual front in Fig. 3 b), it is necessary to first extract and link independent MSM pixels into continuous contours. The algorithm implemented for this purpose scans the MSM edge map (Fig. 3.b)) until a MSM pixel is found. That pixel $(\operatorname{MSM}(i, j))$ becomes the 1st pixel of the nth contour. The algorithm then adds the adjacent pixels by examining the 8-connected neighbors of pixel $\operatorname{MSM}(i, j)$. The contour is terminated when none of the 8-connected MSM pixels neighbors are found.

In an effort to discard short, possibly insignificant fronts, a minimum length criteria is imposed on each extracted front: each edge not exceeding a minimum segment length (10 pixels) is discarded (Oram et al. 2008). Fig. 3 (c) shows the upwelling fronts that are linked and extracted from the MSM pixels map in Fig. 33 (b).

Fig. 4 a) and (b) illustrate the results of upwelling fronts detected with the proposed algorithm, in which the fronts are automatically overlaid on the top of the respectively original SST images in Fig. 2(a) and (b).

\section{Results analysis on the whole database}

In order to validate our algorithm in terms of over-detection or missing-detection of upwelling fronts, we compared our results to the algorithm defined in Oram et al. (2008). The latter extended the standard Canny edge detector (Canny, 1986) by using an automatic selection of the hysteresis thresholds values from the global cumulative histogram in each image. The main advantages of the Oram et al. (2008) algorithm are: 1) the use of the Canny edge detector, which is a well-known and wellestablished gradient-based edge detector; and 2) the fact that the Canny edge detector is used as comparative method with a recent developed algorithm (Nieto et al., 2012). Fig. 5 shows the results of the Oram et al. (2008) method (black contours) overlaid on the original images in Fig. 2

It is necessary to mention that, the in-situ measurements, e.g., buoys data, can give us a sounder support to the results obtained by the proposed approach. However, these measures are very complex to establish and would be extremely challenging for a use in coastal zone context, because of lack of these measures for all the SST satellite images used in this study and for every specific region of the study area. In this sense, it is a well-known fact that validation in the case of ocean data is often and truly performed by assessing the results by professional oceanographers (Nascimento et al. 2012; Tamim et al., 2013.
Sousa et al. 2008), which has been done in the case of this work.

Throughout this evaluation, we used 5 grades: "Bad", "Poor", "Acceptable", "Good" and "Excellent". "Bad" is assigned when the upwelling fronts are not well detected by the algorithms (MSM or Oram et al. (2008)), and "Excellent" is assigned when all the thermal fronts are well identified.

Regarding the results of the evaluation presented in Fig. 6. we can conclude that the MSM method outperforms the Oram et al. (2008) method which is based on the Canny edge detector with automatic selection of the hysteresis thresholds values. Indeed, for the grade "Bad", the value of $2 \%$ is reached by our proposed method while for the Oram et al. (2008) the value of 9\% is attained. For the grade "Good" a value of $48 \%$ is achieved using the MSM method and $40 \%$ with Oram et al. (2008). Overall, $54 \%$ and $42 \%$ are reached by the two grades "Good" and "Excellent" together respectively for MSM and Oram et al. (2008) methods.

After this evaluation of the two fronts detection methods, over this representative database, we can conclude that the proposed algorithm using the MSM method has provided a satisfactory results and shown to be superior to that of the method used in Oram et al. (2008). In fact, the MSM method detects almost all the thermal fronts in SST images and has the advantage of remove all the detected contours in the cloud contamination regions. However, the method used in Oram et al. (2008) does not detect all the real fronts (Fig. 6) and has the limitation to misclassify the cloud contamination regions as frontal regions (Fig. 5(a) and (b)), namely in the area near shoreline and islands. More importantly, the fronts detection quality obtained by this method suggests that it can be used to obtain a first approximation of the upwelling fronts and serve as a basis for a further and subsequent steps in the analysis of the SST images of the ocean.



Fig. 6: Qualitative evaluation made by the oceanographer of fronts detection results produced by MSM and Oram et al. (2008) methods applied over the 92 SST images. 


\section{Conclusion and future prospects}

The singularity exponents computed in a microcanonical formulation show to be an effective approach for the identification and detection of upwelling fronts and filaments in the Moroccan coastal upwelling. The implemented methodology starts by detecting the mesoscale structures in the SST images using the MSM method, followed by an extraction process in order to link the individual edge pixels. The work reported in this paper presents the first and the basis step in the analysis of the SST images. The next step will include the automated classifying of the thermal structures, delineated previously, in order to indicate their association with some various oceanographic structures, such as fronts, filaments and eddies characterizing the variability of the Moroccan surface ocean. The developed algorithms have been successfully applied over a database of 92 AVHRR daily SST images, covering the southern part of Moroccan Atlantic coast.

\section{Acknowledgement}

This work is funded by the French-Moroccan research program Volubilis (MA/11/256) and the project $\mathrm{n}^{\circ}$ MPI 12/2010.

\section{References}

Atillah, A., Orbi, A., Hilmi, K., Mangin, A., 2005. Produits opérationnels d'océanographie spatiale pour le suivi et l'analyse du phénomène d'upwelling Marocain. Geo Observateur 14, 49-62.

Bakun, A., 2006. Fronts and eddies as key structures in the habitat of marine fish larvae : Opportunity, adaptative response and competitive advantage. Scientia Marina 70, 105-122.

Belkin, I.M., O'Reilly, J.E., 2009. An algorithm for oceanic front detection in chlorophyll and SST satellite imagery. Journal of Marine Systems 78, 319 326.

Canny, 1986. A computational approach to edge detection. IEEE Transactions on Pattern Analysis and Machine Intelligence 8, 679-698.

Cayula, J.F., Cornillon, P., 1992. Edge detection algorithm for SST images. Journal of Atmospheric and Oceanic Techology 9, 67-80.

Chassot, E., Bonhommeau, S., Reygondeau, G., Nieto, K., Polovina, J.J., Huret, M., Dulvy, N.K., Demarcq, H., 2011. Satellite remote sensing for an ecosystem approach to fisheries management. ICES Journal of Marine Science: Journal du Conseil 68, 651-666.

Frisch, U., 1995. Turbulence : The legacy of A. N. Kolmogorov. Cambridge University Press .

Holyer, R.J., Peckinpaugh, S.H., 1989. Edge detection applied to satellite imagery of the oceans. IEEE Transactions on Geoscience and Remote Sensing 27, 46-56.

Isern-Fontanet, J., Turiel, A., Garca-Ladona, E., Font, J., 2007. Microcanonical multifractal formalism: Application to the estimation of ocean surface velocities. Journal of Geophysical Research: Oceans 112, n/a-n/a.

Maji, S.K., Yahia, H.M., 2014. Edges, transitions and criticality. Pattern Recognition 47, $2104-2115$.

Marcello, J., Marques, F., Eugenio, F., 2005. Automatic tool for the precise detection of upwelling and filaments in remote sensing imagery. Geoscience and Remote Sensing, IEEE Transactions on 43, 1605-1616.

McClain, E.P., Pichel, W.G., Walton, C.C., 1985. Comparative performance of avhrr-based multichannel sea surface temperatures. Journal of Geophysical Research: Oceans 90, 11587-11601.

Metzner, M., Hennings, I., 1995. Interpretation of dynamical processes in the upwelling area off northwest africa using simulated and remotely sensed data, in: Geoscience and Remote Sensing Symposium, pp. 1101-1103.

Nascimento, S., Franco, P., Sousa, F., Dias, J., Neves, F., 2012. Automated computational delimitation of SST upwelling areas using fuzzy clustering. Computers and Geosciences 43, 207-216.
Nieto, K., Demarcq, H., McClatchie, S., 2012. Mesoscale frontal structures in the canary upwelling system: New front and filament detection algorithms applied to spatial and temporal patterns. Remote Sensing of Environment $123,339-346$

Oram, J.J., McWilliams, J.C., Stolzenbach, K.D., 2008. Gradient-based edge detection and feature classification of sea-surface images of the southern california bight. Remote Sensing of Environment 112, 2397 - 2415.

Pont, O., Turiel, A., Yahia, H., 2013. Singularity analysis of digital signals through the evaluation of their Unpredictable Point Manifold. International Journal of Computer Mathematics 90, 1693-1707. URL: https://hal. inria.fr/hal-00688715 doi 10.1080/00207160.2012.748895

Sousa, F.M., Nascimento, S., Casimiro, H., Boutov, D., 2008. Identification of upwelling areas on sea surface temperature images using fuzzy clustering. Remote Sensing of Environment 112, 2817-2823.

Stowe, L.L., Davis, P.A., McClain, E.P., 1999. Scientific basis and initial evaluation of the CLAVR-1 global clear/cloud classification algorithm for the advanced very high resolution radiometer. Journal of Atmospheric and Oceanic Technology 16, 656.

Tamim, A., Minaoui, K., Daoudi, K., Yahia, H., Atillah, A., Smiej, M., Aboutajdine, D., 2013. A simple and efficient approach for coarse segmentation of moroccan coastal upwelling, in: Signal Processing Conference (EUSIPCO), 2013 Proceedings of the 21st European, pp. 1-5.

Turiel, A., Perez-Vicente, C., Grazzini, J., 2006. Numerical methods for the estimation of multifractal singularity spectra on sampled data : A comparative study. Journal of Computational Physics, Elsevier 216, 362 - 390.

Turiel, A., Perez-Vicente, C., Grazzini, J., 2008a. Method and system for the singularity analysis of digital signals. Patent: pct/es2008/070195 .

Turiel, A., Yahia, H., Perez-Vicente, C., 2008b. Microcanonical multifractal formalism : a geometrical approach to multifractal systems. Part I : Singularity analysis. Journal of Physics A : Mathematical and Theoretical 41, 015501-015536.

Walton, C.C., Pichel, W.G., Sapper, J.F., May, D.A., 1998. The development and operational application of nonlinear algorithms for the measurement of sea surface temperatures with the noaa polar-orbiting environmental satellites. Journal of Geophysical Research: Oceans 103, 27999-28012. 